\title{
LEVANTAMENTO DAS ESPÉCIES BOTÂNICAS ASSOCIADAS À SAÚDE BUCAL NO MUNICÍPIO DE SANTA FILOMENA-PE
}

\author{
SURVEY OF BOTANICAL SPECIES ASSOCIATED WITH ORAL HEALTH IN THE MUNICIPALITY OF \\ SANTA FILOMENA-PE
}

DOI: $10.16891 / 2317-434 X . v 8 . e 2 . a 2020 . p p 499-508$

Recebido em: 24.09.2019 | Aceito em: 07.04.2020

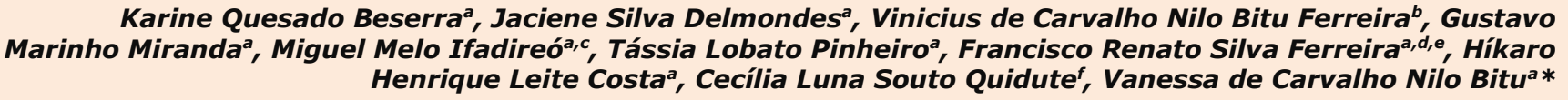

UNILEÃOa
UNEMAT
UPE $^{c}$
FAP $^{d}$
UNIFIP
Faculdade de Medicina de Olinda
E-mail: vanessanilobitu@gmail.com

\section{RESUMO}

As plantas medicinais são usadas empiricamente no tratamento de diversas doenças, porém os usuários não conhecem suficientemente seus efeitos adversos e contraindicações, pautados na ideia de que por serem recursos naturais sejam inofensivas. O presente estudo teve por objetivo realizar um levantamento etnofarmacológico envolvendo o conhecimento dos moradores no município de Santa Filomena-PE na comunidade rural Serra do Inácio. A pesquisa foi realizada através da técnica de curva-espécie-área, respeitando as normas que regulam pesquisas em seres humanos. Foram aplicadas ao público alvo, composto por 31 informantes, entrevistas semiestruturadas. O levantamento dos dados permitiu o registro de vinte e três espécies botânicas diferentes associadas à saúde bucal, pertencentes a dezesseis famílias botânicas, sendo a mais prevalente a Fabaceae. A planta com maior número de citações foi o Ziziphus joazeiro (Juá) e a folha foi a parte das plantas mais utilizada. As principais indicações de uso foram: dor de dente, inflamação e clareamento dentário. Alguns informantes destacaram a importância do horário de coleta das plantas para o alcance do efeito desejado; mas muito pouco foi relatado sobre quantidade necessária para o preparo, contraindicações de uso e efeitos adversos. O conhecimento popular dessa comunidade foi confrontado com dados da literatura pertinente e ficou demonstrado que saberes advindos do povo não devem ser descartados por preconceito acadêmico, uma vez que fornecem informações que podem servir de subsídios para pesquisas laboratoriais futuras.

Palavras-chave: Etnofarmacologia; Plantas medicinais; Odontologia.

\section{ABSTRACT}

Medicinal plants are used empirically in the treatment of various diseases, but users are not sufficiently aware of its adverse effects and contraindications, based on the idea that natural resources are harmless. The present study aimed to carry out an ethno-pharmacological survey involving the residents' knowledge in the city of Santa Filomena$\mathrm{PE}$ in the rural community of Serra do Inácio. The research was performed using the curve-species-area technique, respecting the norms that regulate research in humans. Thirty-one informants were interviewed with semistructured interviews. Data collection allowed the registration of twenty - three different botanical species associated with oral health, belonging to sixteen botanical families, the most prevalent being Fabaceae. The plant with the highest number of citations was Ziziphus joazeiro (Juá) and the leaf was the most used part of the plants. The main indications of use were: toothache, inflammation and tooth whitening. Some informants stressed the importance of the time of collection of the plants to achieve the desired effect; but very little was reported about the amount needed for the preparation, contraindications for use and adverse effects. The popular knowledge of this community was confronted with data from the pertinent literature and it was demonstrated that knowledge from the people should not be discarded due to academic prejudice, since it provides information that can be used as a subsidy for future laboratory research.

Keyword: Ethnopharmacology; Medicinal plants; Dentistry. 


\section{INTRODUÇÃO}

As plantas medicinais vêm sendo usadas de forma terapêutica proporcionando uma melhoria na saúde do indivíduo de forma natural, além de apresentar baixo custo, outros fatores que incrementam a utilização desse recurso natural pela sociedade é a fácil obtenção, pois podem ser cultivadas em quintais, colhidas no entorno das residências por moradores da zona rural ou compradas em mercados e feiras livres (ALBUQUERQUE; MONTEIRO; RAMOS; AMORIN, 2007; OMENA, 2007; BITU et al., 2015). Além desses fatores que fortalecem o uso medicinal de espécies botânicas, estudos de Veiga Jr. e Mello (2008) apontam para o consumismo exacerbado de espécies vegetais para emagrecimento, controle da ansiedade e distúrbios de potência sexual.

O elevado número de espécies vegetais encontradas no Brasil é um fator que contribui para uma grande utilização da fitoterapia no país, pois como possui a possibilidade de usar vários tipos de espécies, tem-se uma maior variabilidade de plantas medicinais que vão ser indicados para cada indivíduo (BORBA; MACEDO, 2006).

Em maio de 2006 o Sistema Único de Saúde-SUS reconhece a prática fitoterápica por meio da portaria ${ }^{\circ} 971$ como a Política Nacional de Práticas Integrativas e Complementares (PNPIC) (SANTOS; DANTAS; SANTOS; DINIZ; SAMPAIO, 2009). Tendo em vista os estudos científicos laboratoriais e essa portaria supracitada, a utilização terapêutica de plantas ganhou legitimidade científica, bem como foram direcionadas as suas indicações de uso, tratando-se de uma política humanizada (FEITOSA; SOARES; BORGES; ANDRADE; COSTA, 2016). De acordo com Pereira; Fernandes e Leite (2012) o Ministério da Saúde instituiu em 2009 o Programa Nacional de Plantas Medicinais e Fitoterápicos (PNPMF), cujo objetivo é que a população tenha acesso seguro e racional das plantas medicinais, além de proporcionar seu uso por meio sustentável da biodiversidade, intensificando a cadeia produtiva e tecnológica de produtos fitoterápicos.

No âmbito odontológico é relatada a utilização de várias espécies de plantas medicinais pouco estudadas (SANTOS; DANTAS; SANTOS; DINIZ; SAMPAIO, 2009; SOUZA; SILVA; MOTA; TORRE; GOMES, 2016). De acordo com Reis et al., (2014) observa-se que, o desconhecimento sobre a fitoterapia pelos cirurgiõesdentistas é o principal fator que limita a prescrição deles nessa área. Fica claro a necessidade de mais trabalhos científicos acerca deste assunto, além da implementação de embasamento teórico sobre fitoterapia na graduação de Odontologia.

De acordo com Oliveira; Mezzomo e Moraes
(2018) a utilização medicamentosa das plantas vem sendo um fato transgeracional. No entanto, muitos usuários não conhecem seus efeitos adversos e as contraindicações, possuindo a ideia que por se tratar de uma "planta" seja algo natural e inofensivo, sendo importante prevenir casos de toxicidade. Essa desconstrução precisa ser realizada através da educação em saúde coletiva, portanto, é necessário investigar sob quais formas o uso empírico é realizado e confrontar os achados com o que está descrito na literatura pertinente sobre efeitos adversos e contraindicações, para que a informação relacionada a utilização terapêutica dos recursos naturais retorne à população de maneira correta, respeitando o consenso na Etnobiologia de que todo e qualquer estudo que envolva conhecimentos populares deve garantir o retorno de informações a quem as forneceu.

$\mathrm{O}$ presente estudo realizou um levantamento etnofarmacológico, a partir do conhecimento dos moradores no município de Santa Filomena-PE sobre a utilização terapêutica das espécies botânicas associadas à saúde bucal, bem como informações sobre modo de preparo, forma de uso, partes da planta utilizada, quantidade, indicações, contraindicações e efeitos adversos.

\section{METODOLOGIA}

A cidade de Santa Filomena-PE está localizada na região de sertão do Pernambuco, a $711 \mathrm{~km}$ da capital Recife. Sua população é de 14.390 habitantes. Localiza-se a uma latitude $08^{\circ} 09^{\prime} 45^{\prime \prime}$ sul e uma longitude $40^{\circ} 36^{\prime} 56^{\prime \prime}$ oeste, estando a uma altitude de 630 metros na unidade geoambiental da depressão sertaneja, sua vegetação predominante é representada pela caatinga, típica da região semiárido, sendo caducifólias (IBGE, 2015).

Os entrevistados deste estudo são os habitantes da comunidade rural Serra do Inácio, município de Santa Filomena-PE. A pesquisa foi realizada através da técnica de curva-espécie-área, assim, foram registradas as espécies informadas a cada entrevista até que essas citações ficaram iguais as que já foram relatadas, estabilizando a curva (ACIESP, 1997).

Os dados foram coletados através de questionários semiestruturados, sendo um estudo observacional descritivo que contém questões norteadoras para permitir atingir os objetivos propostos. A entrevista semiestruturada foi escolhida pela necessidade de deixar o informante à vontade para desenvolver a sua fala, sem perder a objetividade (AMOROZO; VIERTLER, 2010).

Por meio do pré-teste, os informantes foram 
questionados sobre dados sociodemográficos e sobre a origem do conhecimento sobre o uso terapêutico das plantas.

Posteriormente, foram levantados dados referentes ao local e horário de coleta das plantas, forma de adquirir, parte da planta utilizada, modo de preparo, quantidade necessária, indicação, contraindicações de uso e efeitos adversos. As informações coletadas foram concluídas pela técnica da lista livre (ALBUQUERQUE; MONTEIRO; RAMOS; AMORIN, 2007). A análise dos dados coletados permite a tabulação de espécies em um quadro que faz a correlação de todas as variáveis pesquisadas (espécie, origem, parte utilizada, modo de preparo, quantidade, indicações e contraindicações). Nesta fase, foram utilizadas técnicas descritas na literatura e comumente utilizadas em trabalhos similares (TROTER; LOGAN, 1986; BENNETT; PRANCE, 2000).

O presente trabalho atendeu as normas que regulam pesquisas em seres humanos, dispostas na resolução $\mathrm{n}^{\circ} 466$ do Ministério da Saúde, de 12 de dezembro de 2012 e foi aprovado pelo Comitê de Ética e Pesquisa do Centro Universitário Dr. Leão Sampaio sob o parecer de número 3.226.529. O uso do questionário semiestruturado apresentou um risco mínimo de constrangimento para os entrevistados, mas foi reduzido através da confidencialidade do nome dos participantes, que foram esclarecidos de que toda e qualquer informação coletada seria utilizada somente para esta pesquisa. O modo de reprodução deste estudo excluiu qualquer possibilidade de risco físico ao entrevistado (PESSALACIA; RIBEIRO, 2011).

\section{RESULTADOS E DISCUSSÃO}

Os dados da pesquisa abrangeram um total de trinta e um informantes de famílias diferentes, os resultados sociodemográficos estão descritos detalhadamente na tabela 1.

Tabela 1. Dados socioeconômicos e demográficos dos participantes da pesquisa.

\begin{tabular}{lc}
\hline & Dados dos Entrevistados \\
\hline Idade & Quantidade \\
20 a 30 & 04 \\
31 a 40 & 07 \\
41 a 50 & 06 \\
51 a 60 & 05 \\
61 a 70 & 06 \\
71 a 80 & 03 \\
Gênero & \\
Masculino & 06 \\
Feminino & 25 \\
Escolaridade & \\
Sem Alfabetização & 06 \\
Fundamental Incompleto & 15 \\
Fundamental Completo & 02 \\
Médio Incompleto & 01 \\
Médio Completo & 03 \\
Superior Completo & 04 \\
Renda & \\
Até 1 salário & 12 \\
acima de 1, até 2 salários & 10 \\
acima de 2, até 3 salários & 09 \\
Participantes por domićlio & \\
2 & 06 \\
3 & 06 \\
4 & 08 \\
5 & 05 \\
Acima de 5 & 06 \\
Uso da Planta Medicinal & \\
Sim & 23 \\
Não & \\
\hline
\end{tabular}


Pais/Avós

Pais/Avós/Prático
30

01

Fonte: Dados da pesquisa, 2019.

Os resultados obtidos na amostra (tabela 1) mostraram que houve predomínio do sexo feminino. Resultados semelhantes foram encontrados no estudo de Araújo et al., (2015) onde as mulheres predominaram como informantes. Oliveira e Lucena (2015) explicam que em regiões localizadas no interior, as mulheres geralmente encontram-se em casa cuidando dos afazeres domésticos. Esse trabalho no lar inclui os cuidados de saúde com suas famílias e o contato com seus ascendentes, o que garante que esse tipo de informação possa fluir de uma geração para outra (OLIVEIRA; OLIVEIRA; ANDRADE, 2010).

Dentre os informantes, 23 pessoas relataram que fazem o uso de plantas medicinais na cavidade bucal, enquanto 8 não as utilizam. Em relação ao conhecimento adquirido dos entrevistados que utilizam as espécies botânicas, 30 aprenderam com os pais e avós e apenas um dos entrevistados relatou que além dos pais e avós, teve oportunidade de aprendizagem com um prático, pessoa conhecida na comunidade por exercer a profissão de odontólogo sem a devida formação acadêmica, portanto ilegalmente.

A idade dos entrevistados variou de 20 a 80 anos, a faixa etária mais prevalente foi entre 31 a 40 anos, em contraste com Oliveira e Lucena, (2015) onde a idade que teve maior prevalência foi de 51-60 anos. Percebe-se hoje em pesquisas de coleta de informações que associam saberes tradicionais a métodos curativos, que os indivíduos mais jovens cada vez menos preocupam-se em aprender e multiplicar conhecimentos sobre plantas utilizadas terapeuticamente em suas comunidades e/ou famílias. Com o passar dos anos, isso pode acarretar uma perda significativa de informações pela falta de registros, o que segundo Amorozo (2002) pode corresponder ao empobrecimento da cultura de um povo.

Em relação ao grau de escolaridade, a classe com maior número de informantes foi a de ensino fundamental incompleto (15), seguido de participantes que não tem alfabetização (6), superior completo (4), médio completo (03), fundamental completo (02) e médio incompleto (01). Pires et al., (2014) obtiveram resultados parecidos onde a maior quantidade possuía $1^{\circ}$ grau incompleto, corroborando também com Caetano; Ferreira; Reis; Neo e Carvalho (2015) que teve lavradores como sujeitos de pesquisa e que por dedicarem grande parcela do seu tempo ao trabalho, não conseguiam conciliar com os estudos. Contudo, resultados divergentes foram encontrados por Oliveira e Lucena, (2015) onde o nível de escolaridade mais prevalente foi o ensino médio completo. Outro ponto interessante em relação a esse tópico é que as pessoas mais escolarizadas também são aquelas que mais facilmente têm acesso aos serviços formais de saúde por isso prescindem do uso empírico de recursos naturais em seus tratamentos curativos (AMOROZO, 2002).

A renda familiar da maior parcela dos informantes foi de até um salário mínimo; trabalhos de Miranda; Souza; Amaro; Rosa e Carvalho (2013) e Moura (2000) demonstram que, por ser de fácil acesso e baixo custo, as plantas cultivadas em quintais e/ou comercializada em mercados e feiras são uma alternativa de tratamento para as camadas menos favorecidas da população.

O levantamento realizado mostrou 23 espécies botânicas associadas à saúde bucal pertencentes a 16 famílias, sendo a mais prevalente a Fabaceae, contendo quatro espécies, seguida de Lamiaceae, Anacardiaceae, Zingiberaceae e Euphorbiaceae com duas espécies cada uma (tabela 2). Estudos de Ribeiro et al, (2014) obtiveram resultados semelhantes, apresentando a Fabaceae como a mais representativa. As famílias Fabaceae e a Lamiaceae também foram referenciadas como as mais representativas em estudo realizado por BITU et al. (2015), a partir do qual foi construído um catálogo contendo 91 espécies vegetais que são comercializadas nos mercados públicos para fins terapêuticos na região do Cariri, estado do Ceará. 
Tabela 2. Informações etnobotânicas sobre plantas medicinais listadas pelos informantes

\begin{tabular}{|c|c|c|c|c|c|c|}
\hline $\begin{array}{c}\text { NOME } \\
\text { VULGAR }\end{array}$ & $\begin{array}{c}\text { NOME } \\
\text { CIENTÍFICO/DADO } \\
\text { S DO ESPÉCIME }\end{array}$ & FAMÍLIA & $\begin{array}{l}\text { FORMA DE } \\
\text { USO }\end{array}$ & $\begin{array}{l}\text { MODO DE } \\
\text { PREPARO }\end{array}$ & $\begin{array}{l}\text { PARTE DA } \\
\text { PLANTA }\end{array}$ & INDICAÇÃO \\
\hline Açafrão & $\begin{array}{c}\text { Curcuma longa } \\
\text { L.(EAC0034810) }\end{array}$ & Zingiberaceae & Tópico & Pó & Caule & $\begin{array}{l}\text { Ferida na boca/Dor } \\
\text { de dente }\end{array}$ \\
\hline Andu & $\begin{array}{l}\text { Cajanus cajan } \\
\text { (L.) Millsp. } \\
\text { (RB00170078) }\end{array}$ & Fabaceae & Bochecho & Chá & Folha & $\begin{array}{c}\text { Dor de } \\
\text { dente/Inflamação }\end{array}$ \\
\hline Aroeira & $\begin{array}{l}\text { Schinus terebinthifolius } \\
\text { Raddi. (EAC0027480) }\end{array}$ & Anacardiaceae & Bochecho & Chá & Caule & Abcesso dentário \\
\hline Arruda & $\begin{array}{l}\text { Ruta graveolens } \\
\text { L. (Arruda). } \\
\text { (EAC0027480) }\end{array}$ & Rutaceae & Tópico & $\begin{array}{l}\text { Amassada e } \\
\text { morna/ } \\
\text { Macerada+ } \\
\text { Aguardente }\end{array}$ & Folha & $\begin{array}{c}\text { Dor de } \\
\text { dente/Inflamação }\end{array}$ \\
\hline Babosa & $\begin{array}{c}\text { Aloe vera } \\
\text { (L.) Burm. } \\
\text { (EAC0054064) }\end{array}$ & Liliaceae & Tópico & Geléia gelada & Folha & $\begin{array}{c}\text { Dor de } \\
\text { dente/Inflamação }\end{array}$ \\
\hline Bálsamo & $\begin{array}{l}\text { Sedum dendroideum } \\
\text { (SD) (ESA115687) }\end{array}$ & Crassulaceae & Tópico & Macerado & Folha & $\begin{array}{c}\text { Dor de } \\
\text { dente/inflamação }\end{array}$ \\
\hline Bananeira & $\begin{array}{c}\text { Musa } \\
\text { spp. (EAC0043631) }\end{array}$ & Musaceae & Tópico & Leite & Caule & Sangramento \\
\hline Batata-doce & $\begin{array}{l}\text { Ipomoea batatas }(\mathrm{L} .) \\
\quad(\text { EAC0031145) }\end{array}$ & Convolvuláceae & Bochecho & Chá & Folha & $\begin{array}{l}\text { Dor de dente/Depois } \\
\text { de uma extração }\end{array}$ \\
\hline Braúna & $\begin{array}{l}\text { Melanoxylon braúna } \\
\text { Schott. } \\
\text { (HUEFS072250) }\end{array}$ & Fabaceae & Tópico & Raspa & Caule & Dor de dente \\
\hline Cajú & $\begin{array}{c}\text { Anacardium } \\
\text { occidentale }(\mathrm{L} .) \\
(\mathrm{EAC} 0001994)\end{array}$ & Anacardiaceae & Bochecho & Molho & Casca & $\begin{array}{c}\text { Dor de } \\
\text { dente/Inflamação }\end{array}$ \\
\hline Canafístula & $\begin{array}{c}\text { Peltophorum dubium } \\
\text { (Spreng.) } \\
\text { (EAC0029429) }\end{array}$ & Fabaceae & $\begin{array}{l}\text { Bochecho/ } \\
\text { Tópico }\end{array}$ & Chá/Raspa & Caule & Dor de dente \\
\hline Gengibre & $\begin{array}{c}\text { Zingiber officinale } \\
\text { (Roscoe)(EAC002399) }\end{array}$ & Zingiberaceae & $\begin{array}{l}\text { Tópico/Masti } \\
\text { gação }\end{array}$ & Raspa & Raiz & $\begin{array}{l}\text { Mau Hálito/Dor de } \\
\text { dente }\end{array}$ \\
\hline Hortelã & $\begin{array}{l}\text { Mentha spp. } \\
\text { (RB00297544) }\end{array}$ & Lamiaceae & $\begin{array}{c}\text { Tópico/ } \\
\text { Mastigação/ } \\
\text { Chá Baitello }\end{array}$ & $\begin{array}{l}\text { Macerado/Chá/ } \\
\text { Macerado+ } \\
\text { Aguardente }\end{array}$ & Folha & $\begin{array}{l}\text { Dor de dente/Ferida } \\
\text { na boca/Inflamação }\end{array}$ \\
\hline Juá & $\begin{array}{c}\text { Ziziphus joazeiro } \\
\text { (Mart.)(RB01415589) }\end{array}$ & Rhamnaceae & Escovação & Raspa & Caule & $\begin{array}{l}\text { Limpeza/Dor de } \\
\text { dente/Inflamação/Cla } \\
\text { rear/ Não criar cárie }\end{array}$ \\
\hline Malva & $\begin{array}{l}\text { Malva sylvestris } \\
\text { (L.)(ASE0036143) }\end{array}$ & Malvaceae & Bochecho & Chá & Raiz & Dor de dente \\
\hline Manjericão & $\begin{array}{l}\text { Ocimum basilicum } \\
\text { (L.)(EAC0000250) }\end{array}$ & Lamiaceae & Tópico & Macerado & Folha & Dor de dente \\
\hline Palma & $\begin{array}{c}\text { Opuntia ficus-indica } \\
\text { (Mill.)(HUEFS013302) }\end{array}$ & Cactaceae & Bochecho & $\begin{array}{l}\text { Cozinha+sal+pi } \\
\text { ão roxo+sabugo } \\
\text { de milho+brasa }\end{array}$ & Folha & $\begin{array}{l}\text { Depois de uma } \\
\text { extração }\end{array}$ \\
\hline Pau de leite & $\begin{array}{l}\text { Sapium glandulosum } \\
\text { (L.)(EAC0026170) }\end{array}$ & Euphorbiaceae & Beber & Chá & Caule & Ferida na boca \\
\hline
\end{tabular}




\section{8, N. 2 (2020) I ISSN 2317-434X}

\begin{tabular}{|c|c|c|c|c|c|c|}
\hline Pau santo & $\begin{array}{c}\text { Bulnesia sarmientoi } \\
\text { (Lorentz ex Griseb.) } \\
\text { (HUEFS152621) }\end{array}$ & Zygophyllaceae & Beber & Chá & Caule & Dor de dente \\
\hline Pião-roxo & $\begin{array}{c}\text { Jatropha gossypiifolia } \\
\text { (L.)(EAC0044639) }\end{array}$ & Euphorbiaceae & Tópico/Beber & $\begin{array}{c}\text { Macerado/Chá+ } \\
\text { aguardente/Chá/ } \\
\text { Macerado+ } \\
\text { aguardente }\end{array}$ & Folha/Caule & $\begin{array}{c}\text { Dor de } \\
\text { dente/Inflamação }\end{array}$ \\
\hline Romã & $\begin{array}{l}\text { Punica granatum } \\
\text { (L.)(EAC0012808) }\end{array}$ & Pumicaceae & $\begin{array}{c}\text { Beber/ } \\
\text { Bochecho }\end{array}$ & Chá/Molho & Casca & $\begin{array}{c}\text { Dor de dente/Ferida } \\
\text { na } \\
\text { boca/Inflamação/Dep } \\
\text { ois de uma extração }\end{array}$ \\
\hline Tamarindo & $\begin{array}{l}\text { Tamarindus indica } \\
\text { (L.)(EAC0056965) }\end{array}$ & Fabaceae & Tópico/Beber & Macerado/Chá & Folha & $\begin{array}{c}\text { Dor de } \\
\text { dente/Inflamação }\end{array}$ \\
\hline Vassourinha & $\begin{array}{c}\text { Scoparia dulcis } \\
\text { (Sweet Broomweed) } \\
\text { (SPF00209479) }\end{array}$ & Plantaginaceae & Tópico & $\begin{array}{l}\text { Macerado+ } \\
\text { cravo+ } \\
\text { Aguardente }\end{array}$ & Folha & $\begin{array}{c}\text { Dor de } \\
\text { dente/Inflamação }\end{array}$ \\
\hline
\end{tabular}

Fonte: Dados da pesquisa, 2019; Reflora - Herbário Virtual, 2020.

A respeito das espécies citadas nesse estudo, a Schinus terebinthifolius (aroeira- EAC0027480) foi indicada pelos nossos informantes para tratar abcessos dentários. Estudos farmacológicos de Ribas et al. (2006) evidenciaram que o Schinus terebinthifolius promove reparação tecidual acelerada. Semelhante resultado foi encontrado por Nunes jr. et al., (2006) que demonstraram histologicamente que possui efeito cicatrizante dessa espécie botânica.

Participantes do presente estudo apontaram o uso tradicional da Aloe vera (babosa- EAC0054064) no tratamento de dor e inflamação no dente. Faleiro; Elias; Cavalcanti e Cavalcanti (2009) demonstraram o efeito cicatrizante dos componentes do Aloe vera (babosa), que também apresenta atividade antiartrítica e antiinflamatória, sendo essa última segundo Ramos e Pimentel (2011) devido a presença do composto antraquinona. No entanto, essa espécie pode ser tóxica se ingerida em doses altas, o que pode causar uma nefrite aguda. Com isso a importância de ser cauteloso durante sua utilização (NASCIMENTO JÚNIOR et al., 2015).

Estudos de Santos et al., (2018) avaliaram as propriedades da casca e folha do Ziziphus joazeiro (JuáRB01415589), que mostraram atividades antimicrobiana, o que revela concordância com o uso etnofarmacológico dessa planta na região pesquisada. A Ruta graveolens (arruda- EAC0027480) em experimentos laboratoriais de Ruíz; Valenzuela; Ruíz; Veja e Flores (2017) mostrou atividades antiparasitárias, antioxidante e antimicrobiana, quando testado o extrato hidro-alcoólico das folhas; fato esse que merece ser salientado porque alguns entrevistados revelaram fazer uso dessa planta macerada com aguardente.

De acordo com Dukić; Božin; Soković; Mihajlović e Matavulj (2003) foi observada atividade antimicrobiana de óleos essenciais de variadas espécies de Mentha (Hortelã- RB00297544) e com isso foi constatado ação antibacteriana e antifúngica; o seu uso pelos entrevistados nessa pesquisa foi citado para tratamento de feridas bucais entre outras indicações conforme tabela 2. Santos et al., (2017) mostrou que a presença de taninos e alcaloides na Punica granatum (Romã- EAC0012808) lhe confere atividade antimicrobiana, além de apresentar também propriedades antioxidantes, potencial anticancerígeno e antiinflamatório explicada pela presença de fenólicos como antocianinas, flavonóides, entre outros, em diferentes partes dessa planta que teve quatro indicações de uso voltadas para a saúde bucal na população estudada.

Outra planta citada pelos entrevistados foi a Jatropha gossypiifolia (Pião-roxo- EAC0044639) que segundo trabalho de Almeida; Araújo; Morales; Iseppon e Vidal (2015) possui propriedades antiiflamatórias, antimicrobianas, analgésicas, inseticidas, antimaláricas, sedativos, antidiarreicos entre outros, utilizando variadas partes das plantas.

Para a população investigada, o pó da Curcuma longa (Açafrão- EAC0034810) pode ser utilizado no tratamento de dor de dente e lesões bucais; sua atividade antiinflamatória já foi investigada através de métodos laboratoriais por Martinez et al., (2017) que também mencionaram que o extrato de rizoma de Açafrão resulta na curcumina (flavanóide natural), responsável pela boa atividade antioxidante, antiinflamatória e antineoplásica.

O Anacardium occidentale (CajuAC0001994) é utilizada pelos entrevistados na forma de bochecho feito a partir da casca com indicação de uso em processos inflamatórios. Esse resultado corrobora com 
Pereira et al., (2015) onde o extrato da casca do cajueiro possui ação antiinflamatória e antimicrobiana devido a presença de taninos. Em outro trabalho realizado por Silva et al. (2007), o cajueiro mostrou-se efetivo a infeccões provocadas Staphylococcus aureus por apresentar em sua composição taninos e alcaloides.

$$
\text { O Zingiber officinale (Gengibre- }
$$

EAC0023994) foi outra planta citada pelos entrevistados, sendo utilizada para mau hálito e dor de dente. No estudo de Ramos; Junior e Andreani (2016) foi mencionado que essa espécie botânica apresenta atividade antifúngica, corroborando com os resultados encontrados na pesquisa.

A Malva sylvestris (Malva- ASE0036143) é utilizada para dor de dente pelos entrevistados. Popularmente é conhecida por possuir atividades antiinflamatórias e antimicrobianas, no entanto mostrase necessário a realização de estudos científicos que comprovem o uso dessa espécie botânica no tratamento das afecções orais (OLIVEIRA et al., 2007).

$\mathrm{O}$ levantamento realizado mostrou que vinte e dois participantes não conhecem efeitos adversos das plantas medicinais por eles utilizadas, apenas uma pessoa relatou que o gengibre possui reação adversa, que seria "dormência na boca". De acordo com Caetano; Ferreira; Reis; Neo e Carvalho (2015), a utilização de espécies botânicas feita sem noções básicas de toxicidade é considerada perigosa, pois tem potencial de promover interações medicamentosas e desencadear efeitos adversos. Souza; Silva; Mota; Torre e Gomes (2016) também salientou que a utilização incorreta das espécies poderá levar a problemas severos, bem como seu desconhecimento sobre as contraindicações ou uso conjuntamente com outros medicamentos, diante disso é de suma importância sobre o conhecimento da utilização segura das espécies botânicas, para evitar danos à saúde.

Em relação as contraindicações do uso de plantas em tratamentos de saúde bucal, só um participante revelou conhecimento, enaltecendo que as gestantes não deveriam fazer uso da arruda. Estudos laboratoriais comprovaram o efeito embriotóxico e teratogênico a partir do extrato aquoso das folhas dessa espécie que possui ainda atividade anti-helmíntica, antihemorrágica, abortiva, carminativa, antiespamódica e estimulante, é indicada para tratar reumatismo, hipertensão e verminoses e sua contraindicação durante o período gestacional decorre de sua capacidade para estimular contrações uterinas o que pode levar ao aborto ou parto prematuro (RODRIGUES et al.,2011; MATOS, 2000; SOUSA; MATOS; MATOS; MACHADO; CRAVEIRO, 2004).

Em relação a quantidade utilizada das espécies botânicas, a maior parte dos entrevistados relatou não possuir padronização sobre a quantidade usada, apenas em uma espécie foi observado essa padronização, a arruda. BITU et al., (2015) obtiveram resultados parecidos em seus estudos realizados em mercados públicos na região do Cariri cearense, pois não havia, por parte dos vendedores, uma preocupação em relação à forma de preparo que era ensinada sem padronização em relação à quantificação de folhas, raízes, sementes, casca e fruto; e sobre toxicidade. A não padronização de quantidades utilizadas de partes da planta já demonstra o desconhecimento sobre o risco de usá-las indiscriminadamente.

Dos entrevistados na comunidade rural da Serra do Inácio, quinze informaram não haver uma padronização para o horário da coleta e a maioria das plantas citadas são obtidas nos quintais das casas. Nesse estudo, os entrevistados relataram oito plantas que deveriam ser coletadas em horas específicas para ser alcançado o efeito por eles desejado: Ziziphus joazeiro (Juá- RB01415589), Mentha (Hortelã- RB00297544), Punica granatum (Romã- EAC0012808), Anacardium occidentale (Cajú- EAC0001994), Bulnesia sarmientoi (Pau Santo- HUEFS152621), Musa (BananeiraEAC0043631), coletadas no horário da manhã; já a Schinus terebinthifolius (Aroeira- EAC0027480) precisa ser coletada à noite e a Opuntia ficus-indica (PalmaHUEFS013302) no final da tarde. O horário de coleta influencia a atividade biológica das plantas pelas diferentes expressões dos seus princípios ativos quando a espécie é exposta ao sol; Bitu et al. (2012) extraíram óleo essencial de Lippia gracillis (Alecrim da chapada) em diferentes horários do dia sendo realizada a prospecção fitoquímica posteriormente, a partir da qual ficou evidenciado que o timol e o carvacrol estão presentes em maior quantidade quando a planta é coletada sob exposição amena à luz solar (início da manhã e final da tarde). Esses resultados sugerem a realização de estudos fitoquímicos que esclareçam a expressão desses compostos nos produtos extraídos de plantas coletadas nos horários referidos por eles durante as entrevistas.

A parte da planta mais utilizada pelos entrevistados são as folhas, resultados semelhantes foram encontrados em estudos de Borba e Macedo (2006) evidenciando que os indivíduos procuram manter a integridade da planta usando partes da mesma que são renováveis sem comprometer a espécie, evitando assim a perda ou extinção.

Quanto a via de administração mais prevalente foi a via tópica, seguida da via oral, especificamente a realização de bochechos. As formas de preparo citadas 
pelos informantes que mais receberam destaques foram os chás (por decocção) e maceração; resultados semelhantes aos encontrados por Almeida Neto; Barros e Silva, (2015). As características das plantas podem ser essenciais na escolha do preparo; na região estudada existem espécies nativas da caatinga e muitas são as cascas disponíveis, constituindo segundo Ribeiro et al. (2014) uma maneira simples e eficaz para o modo de preparo.

\section{CONCLUSÃO}

Os saberes tradicionais estão presentes na cultura de um povo e de fato, são repassados oralmente de uma geração para outra. No entanto, percebemos durante a realização deste trabalho, o quanto os indivíduos mais jovens estão se distanciando dessas práticas curativas não-formais ao mesmo tempo em que alguns autores do espaço acadêmico, alunos e até professores, encaram os conhecimentos tradicionais de forma preconceituosa.

Diante dessa perspectiva, o registro dessas informações constitui uma fonte de ideias a serem exploradas em futuros estudos bioprospectivos com diferentes abordagens que visem a descoberta de novos fármacos, mesmo não sendo esse o único fundamento de trabalhos etnofarmacológicos. Do ponto de vista da Antropologia e da Sociologia, esses saberes são válidos e devem ser respeitados por constituírem parte da cultura de um povo ou de uma região, mesmo quando os estudos laboratoriais não corroborem com a indicação de uso empírico.

\section{REFERÊNCIAS}

ACADEMIA DE CIÊNCIAS DO ESTADO DE SÃO PAULO-ACIESP. Glossário de Ecologia. 2ed. São Paulo: ACIESP, 1997.

ALBUQUERQUE, U. P.; MONTEIRO, J. M.; RAMOS, A. A.; AMORIN, E. L. C.; Medicinal and magic plants from a public market in Northeastern Brazil. Journal of Ethnophamacology. v. 110, n. 1, 2007.

ALMEIDA NETO, J. R. de; BARROS, R. F. M. de; SILVA, P. R. R.; Uso de plantas medicinais em comunidades rurais da Serra do Passa-Tempo, estado do Piauí, Nordeste do Brasil. R. bras. Bioci., Porto Alegre, v. 13, n. 3, jul./set. 2015. p. $165-175$.

ALMEIDA, P. M. de; ARAÚJO, S. de S.; MORALES, M. A. M.; ISEPPON, A. M. B.; VIDAL, A. C. B.; Potencial genotóxico do látex do pinhão-manso (Jatropha gossypiifolia L.). Genet Mol. Biol.; Ribeirão Preto, v.38, n.1, Jan./Mar., 2015.

AMOROZO, M. C. M.; VIERTLER, R. B. A abordagem qualitativa na coleta e análise de dados em etnobiologia e etnoecologia. In: ALBUQUERQUE, U. P.; LUCENA, R. F. P.; CUNHA, L. V. F. C.(). Métodos e Técnicas na Pesquisa Etnobiológica e Etnoecológica. [S.1.]: NUPPEA, Série: Estudos e Avanços, 2010.

AMOROZO, M. C. de M.; Uso e diversidade de plantas medicinais em Santo Antonio do Leverger, MT, Acta Bot. Bras.; São Paulo, v. 16, n. 2, Apr. 2002. p. 189-203.

ARAÚJO, M. S. C. de; COSTA, J. W. da; COSTA, A. A. da;
TOCCHIO, P. S. P. de L.; ARAÚJO, L. S. de A.; NUNES, V. M. de A.; A utilização de plantas medicinais e da fitoterapia em comunidades assistidas pela Estratégia Saúde da Família. Rev. Bras. Pesq. Saúde, Vitória, v. 17, n. 4, 2015, p. 6-16.

BENNETT, B. C.; PRANCE, G. T.; Introduced plants in the indigenous pharmacopoeia of Northern South America. Economic Botany, v.54, n.1, 2000.

BITU, V. de C. N.; BOTELHO, M. A; COSTA, J. G. M. da; RODRIGUES, F. F. G; VERAS, H. N. H; MARTINS, K. T., LYRA, A., COLUCHI, G.G., RUELA, R. S., QUEIROZ, D. B., DE SOUZA, J. S; QUINTANS JUNIOR, L. J.; Phythochemical screening and antimicrobial activity of Q9 essential oil from Lippia gracillis. Rev. Bras. Farmacogn. v.22, n.1, 2012. p. 69-75.

BITU, V. de C. N.; BITU, V. de C. N.; MATIAS, E. F. F.; LIMA, W. P. de; PORTELO, A. da C.; COUTINHO, H. D. M.; MENEZES, I. R. A. de; Ethnopharmacological study of plants sold for therapeutic purposes in public markets in Northeast Brazil. Elsevier. Jun.2015.

BORBA, A. M.; MACEDO, M.; Plantas medicinais usadas para a saúde bucal pela comunidade do bairro Santa Cruz, Chapada dos Guimarães, MT, Brasil. Acta Botanica Brasilica, v.20, n.4. 2006. p. 771-782.

CAETANO, N.L.B.; FERREIRA, T.F.; REIS, M.R.O.; NEO, M.R.O.; CARVALHO, A.A. Plantas medicinais utilizadas pela população do município de Lagarto- SE, Brasil - ênfase em pacientes oncológicos. Rev. bras. plantas med., 
Botucatu, v.17, n.4, supl.1, 2015.

DUKIĆ, N. M.; BOŽIN, B.; SOKOVIĆ, M.; MIHAJLOVIĆ, B.; MATAVULJ, M.; Atividades Antimicrobianas e Antioxidantes de Óleos Essenciais de Três Espécies Mentha. Planta Med., v.69, n.5. 2003. p. 413-419.

FALEIRO, C.C.; ELIAS ,S. T. H.; CAVALCANTI, L. C.; CAVALCANTI, Á. S. S.; O extrato das folhas de babosa, Aloe vera na cicatrização de feridas experimentais em pele de ratos, num ensaio controlado por placebo. Natureza on line, v.7, n.2, 2009. p.56-60.

FEITOSA, M. H. A.; SOARES, L. L.; BORGES, G. A.; ANDRADE, M. M.; COSTA, S. de M.; Inserção do Conteúdo Fitoterapia em Cursos da Área de Saúde, Rev. bras. educ. med, Rio de Janeiro, v. 40, n.2, Jun, 2016. p.197 - 203.

INSTITUTO BRASILEIRO DE GEOGRAFIA ESTATISTICA - IBGE. Cidades, 2015. Acessado dia 31 de Outubro de 2018. Disponível em: $<$ https://cidades.ibge.gov.br/brasil/pe/santa-

filomena/panorama>.

MARTINEZ, C. A. Real; OLIVEIRA, V. P. de; CAMPOS, F. G.; KANNO, D. T.; FERNANDEZ, O. O. A.; KADRI, C. J.; PEREIRA, J. A.; Influência da aplicação de enemas com extrato oleoso de curcumina (Curcuma longa) sobre o conteúdo tecidual de mucinas neutras e ácidas na mucosa cólica sem trânsito fecal. J. Coloproctol.; Rio de Janeiro v.37, n.4, , out./dez., 2017.

MATOS, F. J. A.; Plantas medicinais: guia de seleção e emprego de plantas usadas em fitoterapia no nordeste do Brasil. 2ed. Fortaleza: Editora UFC, 2000, 344p.

MIRANDA, G. S.; SOUZA, S. R.; AMARO, M. de O. F.; ROSA, M. B. da; CARVALHO, C. A. de; Avaliação do conhecimento etnofarmacológico da população de Teixeiras - MG, Brasil. Rev Ciênc Farm Básica Apl., v.34, n.4. 2013. p.559-563. ISSN 1808-4532.

MOURA, M. A. D.; Estudo com plantas medicinais mais utilizadas em obstetrícia pela população feminina dos municípios de Itapecerica da Serra e Embu Guaçu - São Paulo. Acta Paulista de Enfermagem, n.13, v.2, 2000. p. 8789.

NASCIMENTO JÚNIOR, B. J.; TÍNEL, L. O.; SILVA, E. S.; RODRIGUES, L. A.; FREITAS, T. O. N.; NUNES, X. P.; AMORIM, E. L. C.; Avaliação do conhecimento e percepção dos profissionais da estratégia de saúde da família sobre o uso de plantas medicinais e fitoterapia em Petrolina-PE, Brasil. Rev. bras. plantas med., Botucatu , v.18, n.1, Mar. 2015. p. p. 57-66.

NUNES JR., J. A. T.; RIBAS FILHO, J. M.; MALAFAIA, O.; CZECZKO, N. G.; INÁCIO, C. M.; NEGRÃO, A; W.; et al.; Avaliação do efeito do extrato hidroalcoólico de Schinus terebinthifolius Raddi (Aroeira) no processo de cicatrização da linea alba de ratos. Acta Cir. Bras., São Paulo, v.21, supl. 3, 2006. p. 8-15.

OLIVEIRA, D. M. S; LUCENA, E. M. P.; O uso de plantas medicinais por moradores de Quixadá-Ceará. Rev. bras. plantas med., Botucatu , v.17, n.3, Sept. 2015. p. 407-412.

OLIVEIRA, F. Q.; GOBIRA, B.; GUIMARÃES, C.; BATISTA, J.; BARRETO, M.; SOUZA, M.; Espécies vegetais indicadas na odontologia. Rev. bras. farmacogn., João Pessoa, v.17, n.3, Julho/Setembro, 2007.

OLIVEIRA, G. L. de; OLIVEIRA, A. F. M. de; ANDRADE, L. de H. C.; Plantas medicinais utilizadas na comunidade urbana de Muribeca, Nordeste do Brasil. Acta bot. Bras., v.24, n.2. 2010. p. 571-577.

OLIVEIRA, V. B. de; MEZZOMO, T. R.; MORAES, E. F. de; Conhecimento e Uso de Plantas Medicinais por Usuários de Unidades Básicas de Saúde na Região de Colombo, PR. Revista Brasileira de Ciências da Saúde. Curitiba, v.22, n. 1, jan. 2018. p.57-64.

OMENA, M. L. R. A. Ensaio Etnofarmacológico de espécies vegetais com ação no Sistema Nervoso Central, Originárias do Bioma Caatinga. Saúde e Ambiente em Revista. v.2, n.2, 2007. p. 92-117.

PEREIRA, A. V.; AZEVÊDO, T. K. B. de; HIGINO, S. S. dos S.; SANTANA, G. M.; TREVISAN, L. F. A.; AZEVEDO, S. S. de; PEREIRA, M. V.; RAMOS DE PAULA, A. F.; Taninos da casca do Cajueiro: atividade antimicrobiana. Revista AGROTEC; v.36, n.1, 2015. p. 121-127.

PEREIRA, F. L.; FERNANDES, J. M.; LEITE, J. P. V.; Ethnopharmacological survey: a selection strategy to identify medicinal plants for a local phytotherapy program. Braz. J. Pharm. Sci., São Paulo, v.48, n.2, June 2012. p. 299-313.

PESSALACIA, J. D.; RIBEIRO, C. R. O.; Entrevistas E Questionários: Uma Análise Bioética Sobre Riscos Em Pesquisa. Ver. Enfermagem do centro Oeste Mineiro, v.1, n.3, 2011.

PIRES, I. F. B.; SOUZA, A. A.; FEITOSA, M. H. A.; COSTA, S. M.; Plantas medicinais como opção terapêutica em comunidade de Montes Claros, Minas Gerais, Brasil. Rev. bras. plantas med., Botucatu , v.16, n.2, supl. 1, 2014. p. 426-433.

RAMOS, A. P.; PIMENTEL, L. C.; Ação da babosa no reparo tecidual e cicatrização. Brazilian Journal of Healt; v.2, n.1, 2011. p.40-48.

RAMOS, K.; JUNIOR, R. A.; ANDREANI, D. I. K.; Óleos 
essenciais e vegetais no controle in vitro de Colletotrichum gloeosporioides. Rev. bras. plantas med., Botucatu, v.18, n.2, supl.1, 2016.

REFLORA. Herbário Virtual. 2020. Disponível em: http://reflora.jbrj.gov.br

REIS, L. B. M. dos; FARIAS, A. de L.; BOLLELLA, A. de P.; SILVA, H. K. M.; CANUTO, M. Í. C.; ZAMBELLI, J. da C.; FREIRE, M. do C. M.; Conhecimentos, atitudes e práticas de Cirurgiões-Dentistas de Anápolis-GO sobre a fitoterapia em Odontologia. Revista de Odontologia da UNESP, Araraquara, v.43 n.5 Set./Out. 2014.

RIBAS, M. de O.; SOUSA, M. H.; SARTORETTO, J.; LANZONI, T. A.; NORONHA, L.; ACRA, L. A.; Efeito da Schinus terebinthifolius raddi sobre o processo de reparo tecidual das lesões ulceradas induzidas na mucosa bucal do rato. Revista Odonto Ciência., Fac. Odonto/PUCRS, v.21, n.53, jul./set. 2006.

RIBEIRO, D. A.; MACÊDO, D. G.; OLIVEIRA, L. G. S.; SARAIVA, M. E.; OLIVEIRA, S. F.; SOUZA, M. M. A.; MENEZES, I. R. A.; Potencial terapêutico e uso de plantas medicinais em uma área de Caatinga no estado do Ceará, nordeste do Brasil. Rev. bras. plantas med., Botucatu, v.16, n.4, Dec. 2014. p. 912-930.

RODRIGUES, H. G.; MEIRELES, C. G.; LIMA, J. T. S.; TOLEDO, G. P.; CARDOSO, J. L.; GOMES, S. L. Efeito embriotóxico, teratogênico e abortivo de plantas medicinais. Rev. Bras. Pl. Med., Botucatu, v.13, n.3, 2011. p.359-366.

RUÍZ, L. D.; VALENZUELA, R. B.; RUÍZ, O. D.; VEJA, M. S.; FLORES, P. G.; Composição química e efeito antibacteriano in vitro de extratos de laria tridentata, origanum vulgare, sagebrush ludovician e ruta graveolens. Nova scientia, León, v.9, n.19, 2017.

SANTOS, E. B.; DANTAS, G. S.; SANTOS, H. B.; DINIZ, M. F. F. Melo; SAMPAIO, F. C.; Estudo etnobotânico de plantas medicinais para problemas bucais no município de João Pessoa, Brasil. Revista Brasileira de Farmacognosia, v.19, n.1, Jan/Mar 2009. p.321-324.

SANTOS, M. G. C. dos; NÓBREGA, D., R. de M.; ARNAUD, R. R.; SANTOS, R. C. dos; GOMES, D. Q. de C.; PEREIRA, J. V.; Punica granatum Linn. prevenção da candidíase oral em pacientes submetidos a tratamento antineoplásico. Rev. odontol. UNESP, Araraquara, v.46, n.1, Jan./Feb., 2017.

SANTOS, M. O.; RIBEIRO, D. A.; MACÊDO, M. J.F.; MACEDO, J. G.F.; LACERDA, M. N. S. de; MACÊDO, M. S..; SOUZA, M. M. A.; Plantas Medicinais: versatilidade e concordância de uso na área de caatinga, Nordeste do Brasil. A. Acad. Bras. Ciênc., Rio de Janeiro, v.90, n.3, julho/setembro, 2018.

SILVA, J. G. da; SOUZA, I. A.; HIGINO, J. S.; SIQUEIRA JUNIOR, J. P.; PEREIRA, J. V.; PEREIRA , M. do S. V.; Atividade antimicrobiana do extrato de Anacardium occidentale Linn. em amostras multiresistentes de Staphylococcus aureus. Rev. bras. farmacogn., João Pessoa , v.17, n.4, Dec. 2007 . p. 572-577.

SOUSA, M. P. de; MATOS, F. J. de A.; MATOS, M. E. O.; MACHADO, M. I. L.; CRAVEIRO, A. A.; Constituintes químicos ativos e propriedades biológicas de plantas medicinais brasileiras. 2ed. Fortaleza: Editora UFC, 2004, $448 \mathrm{p}$.

SOUZA, G. F. M. de; SILVA, M. R. A. da; MOTA, E. T. da; TORRE, A. M. L.; GOMES, J. P.; Plantas medicinais $\mathrm{x}$ raizeiros: uso na odontologia. Rev. cir. traumatol. bucomaxilo-fac., Camaragibe, v.16, n.3, set. 2016.

TROTER, R.; LOGAN, M.; Informant consensus: A new approach for identifying potencially effective medicinal plants. Indigenous medicine and diet: biobehavioural approaches, New York, v.1, n.1, 1986.

VEIGA JUNIOR, V. F.; MELLO, J. C. P.; As monografias sobre plantas medicinais. Rev. bras. farmacogn., João Pessoa, v.18, n.3, Sept. 2008. p. 464-471. 